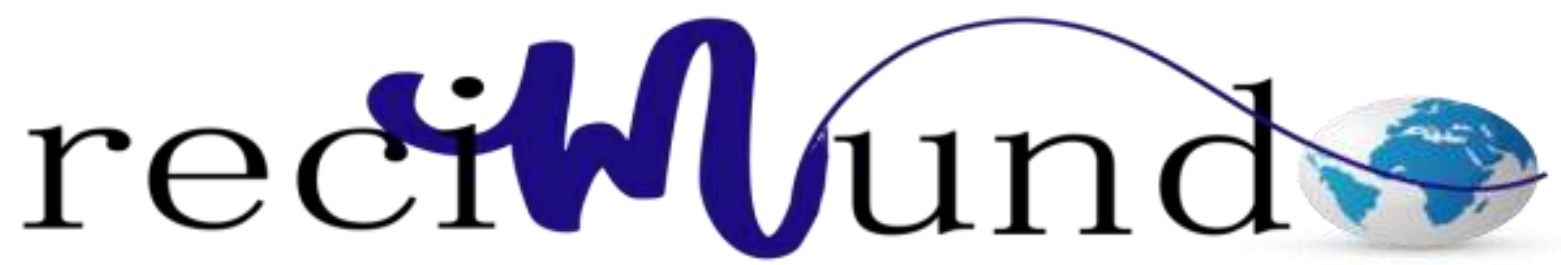

Revista Cientifica Mundo de la Investigación y el Conocimiento

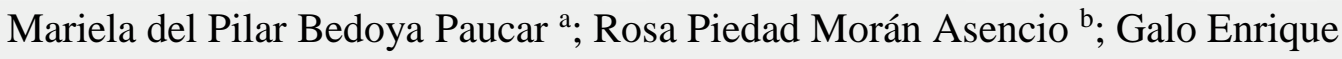 \\ Estupiñan Vera ${ }^{\mathrm{c}}$.
}

Nivel nutricional y régimen alimenticio de pacientes con insuficiencia renal crónica de un centro de diálisis de Chile

Nutritional level and food regime of patients with chronic renal insufficiency of a Chile dialysis center

\begin{abstract}
Revista Científica Mundo de la Investigación y el Conocimiento. Vol. 3 núm.3, septiembre, ISSN: 2588-073X, 2019, pp. 243-274
\end{abstract}

DOI: $10.26820 /$ recimundo/3.(3).septiembre.2019.243-274

URL: $\underline{\text { http://recimundo.com/index.php/es/article/view/520 }}$

Código UNESCO: 3205 Medicina Interna

Tipo de Investigación: Artículo de Revisión

\author{
Editorial Saberes del Conocimiento
}

Recibido: 15/05/2019

Aceptado: 23/06/2019

Publicado: 30/09/2019

Correspondencia: mariela.bedoyap@ug.edu.ec

a. Magister en Enfermería, Licenciada en Enfermería; Universidad de Guayaquil; Guayaquil, Ecuador; mariela.bedoyap@ug.edu.ec

b. Especialista en Enfermería en Cuidados Críticos, Licenciada en Enfermería; Universidad de Guayaquil; Guayaquil, Ecuador; rosa.moranas@ug.edu.ec

c. Magister en Sistemas Integrados de Gestión, Químico y Farmacéutico, Tecnólogo Pedagógico en Informática; Universidad de Guayaquil; Guayaquil, Ecuador; galo.estupinanv@ug.edu.ec 


\section{Nivel nutricional y régimen alimenticio de pacientes con insuficiencia renal crónica de un centro de diálisis de Chile}

Vol. 3, núm. 3., (2019)

Mariela del Pilar Bedoya Paucar; Rosa Piedad Morán Asencio; Galo Enrique Estupiñan Vera.

\section{RESUMEN}

Uno de los principales aparatos funcionales del organismo humano es el urinario. Este se encarga de tomar las sustancias principales de los alimentos y expulsar los desechos a través de la orina. Uno de estos organismos son los riñones que se encargan de filtrar la sangre, regular la presión arterial y el equilibrio ácido/base. Al no tener un buen control de este procedimiento se empieza a padecer de daño renal que se manifiesta en diferentes enfermedades a través de la cantidad y frecuencia de segregación de orina por este organismo. Por ello, cuando los riñones dejan de funcionar se habla de insuficiencia renal crónica, la cual, hoy es un problema de salud pública que tiende a tomar dimensiones de epidemia y tiene un grave impacto en la calidad de la vida del paciente y garantizarle el desarrollo de sus actividades cotidianas adecuadamente, debe recibir tratamiento mediante un proceso de diálisis que le permite eliminar los líquidos y sustancias de desecho del organismo. Ante esto, le corresponde al paciente tener una alimentación balanceada que le ayude a mejorar integralmente, pues, al tener una dieta inapropiada aumenta el número de complicaciones y conduce a su deterioro progresivo. Ante esto, las unidades de diálisis establecidas en Chile, tienen como objetivo que el paciente alcance un estado nutricional adecuado y le permita controlar la azoemia, con el fin de reducir sus efectos para así retrasar la progresión de la insuficiencia renal. En consecuencia, su régimen alimenticio debe ser equilibrado, variado, adecuado en energía, ordenado, adaptado y complementado que les garantice un nivel nutricional funcional a las exigencias del organismo para cumplir con sus tareas. La metodología del artículo en curso, corresponde a la investigación de tipo documental, recogida la información de diferentes fuentes bibliográficas disponibles en medios electrónicos y llegar a las conclusiones.

Palabras Claves: Insuficiencia renal; Diálisis; Nivel nutricional; Régimen alimenticio. 


\title{
Nivel nutricional y régimen alimenticio de pacientes con insuficiencia renal crónica de un centro de diálisis de Chile
}

Vol. 3, núm. 3., (2019)

Mariela del Pilar Bedoya Paucar; Rosa Piedad Morán Asencio; Galo Enrique Estupiñan Vera.

\begin{abstract}
One of the main functional devices of the human organism is the urinary tract. It takes care of taking the main substances from the food and expelling the waste through the urine. One of these organisms is the kidneys that filter blood, regulate blood pressure and acid/base balance. By not having a good control of this procedure, you begin to suffer from kidney damage that manifests in different diseases through the amount and frequency of urine segregation by this organism. Therefore, when the kidneys stop working there is talk of chronic kidney failure, which, today, is a public health problem that tends to take epidemic dimensions and has a serious impact on the quality of life of the patient and guarantee the development of their act adequately, you should be treated through a dialysis process that allows you to remove fluids and waste substances from your body. Faced with this, it is up to the patient to have a balanced diet that helps him to improve fully, because, having an inappropriate diet increases the number of complications and leads to their progressive deterioration. In view of this, the dialysis units established in Chile, aim to reach an adequate nutritional status and allow them to control azoemia, in order to reduce its effects in order to delay the progression of renal failure. Consequently, its diet must be balanced, varied, and adequate in energy, ordered, adapted and supplemented to ensure a functional nutritional level to the requirements of the body to carry out its tasks. The methodology of the article in progress corresponds to the research of documentary type, collected the information from different bibliographic sources available in electronic means and reach the conclusions.
\end{abstract}

Key Words: Renal failure; Dialysis; Nutritional level; Food regimen. 


\section{Nivel nutricional y régimen alimenticio de pacientes con insuficiencia renal crónica de un centro de diálisis de Chile}

Vol. 3, núm. 3., (2019)

Mariela del Pilar Bedoya Paucar; Rosa Piedad Morán Asencio; Galo Enrique Estupiñan Vera.

\section{Introducción.}

Al hacer referencia a la insuficiencia renal, se entiende que es una enfermedad crónica reconocida recientemente como un problema de salud pública global, por su carácter epidémico y complicaciones devastadoras que produce. En Chile el número de pacientes en diálisis crónica (una terapia de sustitución renal de alto costo), ha experimentado un aumento de más de 30 veces en los últimos 25 años. Estos pacientes habitualmente emergen de una población mayor con enfermedades renales crónica, cuya prevalencia se estima en $10 \%$.

No obstante, se hace común, la información disponible acerca de las enfermedades renales crónicas en etapas previas a diálisis es escasa, pues, permanece como una enfermedad subdiagnosticada y de referencia tardía. Su falta de reconocimiento precoz produce consecuencias, la declinación de la función renal se asocia directamente a la acumulación de complicaciones, que devienen en un pronóstico adverso. Durante su evolución silenciosa estas enfermedades, el paciente puede experimentar progresión renal y morbimortalidad cardiovascular. Estudios recientes muestran que la probabilidad de que el paciente con ERC fallezca de complicaciones cardiovasculares es mucho mayor que la progresión a falla renal terminal.

Al respecto, Flores, Alvo, Borja, Morales, Vega y otros (2015), definen a las enfermedades crónicas renales como aquellas caracterizadas por tener

Una velocidad de filtración Glomerular (VFG) $<60 \mathrm{~mL} / \mathrm{mln} / 1,73 \mathrm{~m} 2$, y/o la presencia de daño renal, independiente de la causa, por 3 meses o más. Una VFG <60 ml/min/1,73 m2 por sí 


\section{Nivel nutricional y régimen alimenticio de pacientes con insuficiencia renal crónica de un centro de diálisis de Chile}

Vol. 3, núm. 3., (2019)

Mariela del Pilar Bedoya Paucar; Rosa Piedad Morán Asencio; Galo Enrique Estupiñan Vera.

sola define las enfermedades, porque implica la pérdida de al menos la mitad de la función renal, lo que ya se asocia a complicaciones. (p.137)

De acuerdo con esta definición, se entiende que toda persona al ser diagnosticado en función a esta velocidad de filtración, presenta una enfermedad renal crónica, de allí, la importancia que las naciones busquen alternativas viables para introducir en los respectivos centros de salud, una atención primaria fundamental, mediante la cual, se logre el diagnóstico previo de la misma, para luego establecer planes de controles que ayuden a los individuos a reconocer posibles acciones que le pueden llevar a tener una insuficiencia renal crónica y en ese sentido, asumir la diálisis como vía para mejorar su calidad de vida.

Por lo tanto, durante la evaluación primaria es relevante que el médico tratante incluya durante el diagnóstico, exija al paciente un examen de orina que haga posible confirmar y cuantificar la proteinuria, debe hacerse una evaluación diagnóstica que incluya historia y examen físico acabado, investiga la presencia de diabetes, hipertensión, enfermedades del colágeno, insuficiencia cardíaca y otras. Debe evaluarse la función renal y realizar un examen de orina completo en búsqueda de alteraciones urinarias (microhematuria, cilindros) que sugieran nefropatía primaria. Pues, el paciente con proteinuria debe ser referido al nefrólogo para elaborar una estrategia de estudio y tratamiento adecuado. Se ha demostrado que la interconsulta nefrológica logra aminorar la velocidad de pérdida de función renal en pacientes con proteinuria.

En esta dirección, Flores, Alvo, Borja, Morales, Vega y otros (ob.cit) precisan que los individuos normales: 


\section{Nivel nutricional y régimen alimenticio de pacientes con insuficiencia renal crónica de un centro de diálisis de Chile}

Vol. 3, núm. 3., (2019)

Mariela del Pilar Bedoya Paucar; Rosa Piedad Morán Asencio; Galo Enrique Estupiñan Vera.

Excretan pequeñas cantidades de proteína en la orina, habitualmente menos de $100 \mathrm{mg} /$ día, que consiste principalmente de albúmina (40\%), globulinas de bajo peso molecular (20\%) y otras proteínas de origen tubular y del tracto urinario (40\%). La proteinuria no es más que la excreción de proteína urinaria mayor de $150 \mathrm{mg} /$ día. (140).

De lo indicado por los autores, se puede decir que a medida que las personas aumentan la excreción de proteína, se convierte en individuos caracterizados por la presencia de una insuficiencia renal crónica, que amerita la valoración dado que, el riñón realiza varias funciones interrelacionadas: regulación del metabolismo hidrosalino y ácido-base, control de la presión arterial, depuración de productos nitrogenados, producción de eritropoyetina, activación de vitamina D, que dependen de la velocidad de filtración glomerular la unidad de evaluación de la función renal.

En función a las ideas anteriores, la presencia de un diagnóstico amplio que garantice al paciente valorar su situación de salud vinculada con la insuficiencia renal, representan un proceso continuo; pues, estos pacientes tratan de aceptar su nueva imagen y adaptar su estilo de vida. En tal sentido, Pérez, Salas, León, y López (2019), indica que el tratamiento de diálisis los pacientes con enfermedad renal deben someterse a diálisis regular, enfrentan dificultades para mantener sus empleos, vida social, flexibilidad financiera, limitación de líquidos y alimentos”. (p. 1077). Es decir, complejidad y naturaleza crónica de las enfermedades afectan la calidad de vida de los pacientes con insuficiencia renal crónica, su salud y reducen sus expectativas de vida. Por lo tanto, necesitan un régimen alimenticio que les permita mantener un nivel nutricional que le apoye durante su tratamiento y al mismo tiempo le facilite sus actividades diarias. 


\section{Nivel nutricional y régimen alimenticio de pacientes con insuficiencia renal crónica de un centro de diálisis de Chile}

Vol. 3, núm. 3., (2019)

Mariela del Pilar Bedoya Paucar; Rosa Piedad Morán Asencio; Galo Enrique Estupiñan Vera.

Cabe destacar que, el paciente con insuficiencia renal crónica en tratamiento con diálisis, suele sufrir cambios psicológicos, sociales, en su estilo de vida y también, que no sólo le afectan a él, sino a toda su familia. Es por este motivo, que es trascendental estudiar el régimen alimenticio, con el fin de ofrecerle un nivel nutricional cónsono a sus propios requerimientos. Tal como lo indica, Sandoval (2019) los pacientes con insuficiencia renal crónica, “deben aprender qué comer y cómo alimentarse para gozar del placer de la comida sin poner en peligro su bienestar y salud". (p.18). En otras palabras, cuando los pacientes con insuficiencia renal crónica, se encuentran en tratamiento de diálisis, deben entender el significado que posee para ellos, mantener una dieta alimenticia caracterizada por la combinación de proteínas, hidratos de carbono, grasas, vitaminas, minerales, agua, entre otros.

De allí, que la respectiva combinación balanceada y adaptada a los requerimientos alimenticios necesitados por los pacientes sometidos a diálisis, como resultado de una insuficiencia renal crónica, deben tener presente la necesidad de establecer un plan encargado de ayudarlos a lograr la respectiva organización de cada uno de los grupos alimenticios y con ello, satisfacer sus necesidades, esto hará posible continuar con sus actividades diarias, laborales y sociales. Pues, va a existir un nivel nutricional equilibrado encargado de proporcionarle mejoras en aquellas posibles complicaciones cardiovasculares, frenar la progresión de la enfermedad, además, hace posible cumplir una dieta apetecible que encaje en la medida de lo posible con su estilo de vida.

Los aspectos citados en párrafos anteriores, permiten destacar su importancia para el desarrollo de este artículo, además, ofrecen nuevos conocimientos pertinentes a los tópicos por analizar y así introducen una proyección de la dinámica investigativa, que hace posible entender 


\section{Nivel nutricional y régimen alimenticio de pacientes con insuficiencia renal crónica de un centro de diálisis de Chile}

Vol. 3, núm. 3., (2019)

Mariela del Pilar Bedoya Paucar; Rosa Piedad Morán Asencio; Galo Enrique Estupiñan Vera.

no sólo la presencia de la insuficiencia renal crónica como una situación de salud pública, sino que abarca un espacio de relevancia, como lo constituye el nivel nutricional que estos pacientes deben tener a fin de garantizarles un régimen alimenticio básico y funcional, a fin de lograr su asistencia al tratamiento de diálisis de manera efectiva para continuar con la realización de cada actividad o tarea propuesta en su vida. Aspectos que, resultan un aporte destacado para la construcción del artículo en curso.

\section{Método.}

Una de las exigencias de todo proceso investigativo encargado de interpretar en forma crítica las características básicas de un problema determinado, es encontrar el método adecuado que le ofrezca condiciones viables para dar continuidad a las etapas subsiguientes que determinan a la investigación para con ello fijar los alcances científicos, insertarlos en el estudio propuesto.

En tal sentido, Ruiz (2017) define al método como "estilos de trabajo particulares de cada disciplina y las formas de investigación que se utilizarán para resolver un problema específico de indagación”. (p.69). Por lo tanto, en el contexto investigativo donde se encuentra insertado el presente artículo, se selecciona el método dialéctico, mediante el cual se puede generar contradicciones de los elementos propios y presente en el problema de estudio.

Al respecto, Uribe (2019) sostiene que en el campo documental "no es sólo descubrir una realidad; sino clasificarla de ella con claridad las estructuras compuestas que la forman”. (p. 65). Es decir, separar todas aquellas percepciones encargadas de proyectar hacia la valoración de la realidad e interpretar sus posibles relaciones para así construir un nuevo hecho investigativo. 


\section{Nivel nutricional y régimen alimenticio de pacientes con insuficiencia renal crónica de un centro de diálisis de Chile}

Vol. 3, núm. 3., (2019)

Mariela del Pilar Bedoya Paucar; Rosa Piedad Morán Asencio; Galo Enrique Estupiñan Vera.

\section{Tipo de Investigación.}

Los elementos inherentes a un problema que reúne condiciones para su exploración e indagación, se convierte en un factor esencial para vincularlo con un tipo de estudio. En este particular, el objetivo del artículo logra fijar una postura haca lo documental, apoyado en Uribe (ob.cit) "permite obtener conocimientos a partir del análisis de datos que han sido recolectados o analizados en otras investigaciones". (p.64).

Según la conceptualización dada por Uribe, se puede deducir que, mediante la ubicación científica bajo lo documental, permitirá revisar e interpretar aquellos aportes previos dados por otros investigadores a fin de cumplir las tareas involucradas en este procedimiento científico, para así elaborar de manera sistemática y organizada las conclusiones generales del artículo.

\section{Fuentes Documentales}

Así como es fundamental tener claridad en cuanto al método y tipo de investigación, se hace determinante encontrar las diferentes fuentes que servirán de apoyo para construir el cuerpo teórico del artículo. Para Ruiz (ob.cit) las técnicas documentales "sirven para darle testimonio fiel a las ideas contenidas en un texto”. (p.56). En otras palabras, su utilización hace posible seguir en forma estructurada la presentación de las ideas contenidas en el análisis científico.

De lo expuesto anteriormente, se valora la importancia de cumplir con la lectura reflexiva inicial como una fuente documental que dará oportunidad a los textos seleccionados llevarlos hacia una reflexión de los mismos a fin de captar su sentido general y proyectar los argumentos hacia el interés investigativo. 


\section{Nivel nutricional y régimen alimenticio de pacientes con insuficiencia renal crónica de un centro de diálisis de Chile}

Vol. 3, núm. 3., (2019)

Mariela del Pilar Bedoya Paucar; Rosa Piedad Morán Asencio; Galo Enrique Estupiñan Vera.

\section{Técnicas de Recolección de Información}

Con base a los argumentos anteriores, se puede acotar que para el desarrollo de este contenido de investigación, se tomará en cuenta su naturaleza metodológica, para así llevar a cabo la selección de técnicas bibliográficas apropiadas para elaborar con exactitud las interpretaciones requeridas. Según Uribe (ob.cit) las técnicas de recolección de información "forman un conjunto de tareas apoyadas en la revisión de contenidos que permiten elaborar nuevos conocimientos. (p. 70).

Al tomar en consideración esta última conceptualización, se puede resaltar que, el investigador parte principalmente de una revisión previa, luego organiza los contenidos de interés desde una visión argumentativa e interpretativa, para describir cada hecho y fijar su posición reflexiva ante cada hecho. Esto les dará la respectiva flexibilidad a las tareas investigativas, para finalmente apoyar el discurso que indicará su actuación vinculante con el tema.

\section{Resultados}

Una vez incorporada cada una de las técnicas consideradas como herramientas para dar la respectiva fundamentación al trabajo, se organizan sus contenidos en función a la necesidad investigativa, lo cual es presentado a continuación bajo criterios descriptivos, encargados de apoyar las reflexiones argumentativas que se requieren en su desarrollo. 


\section{Nivel nutricional y régimen alimenticio de pacientes con insuficiencia renal crónica de un centro de diálisis de Chile}

Vol. 3, núm. 3., (2019)

Mariela del Pilar Bedoya Paucar; Rosa Piedad Morán Asencio; Galo Enrique Estupiñan Vera.

\section{Insuficiencia Renal}

La Insuficiencia Renal Crónica Terminal somete al paciente a múltiples limitaciones, sin lugar a dudas la capacidad de trabajar es una de éstas, a lo que se agrega en nuestro país la realidad socioeconómica, que combina ausencia de soporte social, altos niveles de desocupación y disminución o carencia de ingresos familiares. Por todo esto es que se ve a diario, una grave afectación en la calidad de vida de los pacientes a través de aspectos tales como estado nutricional, fallas en la autoestima, depresión, imposibilidad de adquirir medicamentos, acceder a estudios complementarios, sostener a sus familias y otras.

En este sentido, Leung 2003 (citado por Pérez, Salas, León, y López ob.cit), la insuficiencia renal crónica (IRC) es una enfermedad irreversible y progresiva donde el cuerpo no puede mantener el equilibrio metabólico y electrolítico resultando en uremia, acidosis metabólica, anemia, desequilibrios electrolíticos y trastornos endocrinos. Sus principales causas son la diabetes, la hipertensión, glomerulonefritis y enfermedad de riñón poliquístico.

Por ello, los patrones de morbilidad y mortalidad por enfermedad en todo el mundo están cambiando, tanto en el mundo desarrollado como en el emergente. En todo el siglo 20, las enfermedades infecciosas fueron la principal causa de muerte y de discapacidad. No obstante, en este siglo, no contagioso, las enfermedades no infecciosas se han convertido en la principal causa de mortalidad y morbilidad en todo el mundo. Este cambio se refleja en el tipo de enfermedades que causan insuficiencia renal crónica y en su presentación y progresión. 


\section{Nivel nutricional y régimen alimenticio de pacientes con insuficiencia renal crónica de un centro de diálisis de Chile}

Vol. 3, núm. 3., (2019)

Mariela del Pilar Bedoya Paucar; Rosa Piedad Morán Asencio; Galo Enrique Estupiñan Vera.

En esta dirección, Pérez, Salas, León, y López (ob.cit) indica que la enfermedad renal crónica (ERC):

Es una condición común en que hay una pérdida de la función renal con el tiempo. La ERC está asociada con mayores riesgos de varias comorbilidades; incluyendo la enfermedad cardiovascular y la insuficiencia renal crónica. La enfermedad renal crónica es un problema de salud pública mundial emergente. (p.1080)

Según lo planteado, se precisa que los pacientes que presentan insuficiencia renal crónica, están direccionado a mantener mayores riesgos donde los cardiovasculares son determinantes, además, debido a la proyección que la misma ha alcanzado en el tiempo, se convierte en una enfermedad de salud pública, por lo cual, cada país, debe asumir de forma precisa y coherente su respectiva intervención oportuna a fin de ofrecerle a la ciudadanía su respectiva consideración.

Por lo tanto, la enfermedad renal crónica significa que sus riñones están dañados y no pueden filtrar la sangre como deberían. Este daño puede ocasionar que los desechos se acumulen en su cuerpo y causen otros problemas que podrían perjudicar su salud. La diabetes y la hipertensión arterial son las causas más comunes de enfermedad renal crónica. Es decir, el daño renal se produce lentamente durante muchos años. Muchas personas no tienen ningún síntoma hasta que la enfermedad renal está muy avanzada. De allí, que mediante los análisis de sangre y orina son la única manera de saber si usted tiene enfermedad renal.

Es importante resaltar que, la enfermedad renal en etapa final, también denominada enfermedad de los riñones en etapa final, se presenta cuando la enfermedad renal crónica (la 


\section{Nivel nutricional y régimen alimenticio de pacientes con insuficiencia renal crónica de un centro de diálisis de Chile}

Vol. 3, núm. 3., (2019)

Mariela del Pilar Bedoya Paucar; Rosa Piedad Morán Asencio; Galo Enrique Estupiñan Vera. pérdida gradual de la función renal) alcanza un estado avanzado. En la enfermedad renal en etapa final, los riñones ya no pueden funcionar como deberían para satisfacer las necesidades del cuerpo. Los riñones filtran los desechos y el exceso de líquidos de la sangre, que luego se excreta en la orina. Cuando los riñones pierden su capacidad de filtrado, se pueden acumular niveles peligrosos de líquidos, electrolitos y desechos en el cuerpo.

De acuerdo con los planteamientos, se precisa que el daño renal, una vez que se produce, no se puede revertir. Las posibles complicaciones pueden afectar a cualquier parte del cuerpo y pueden incluir: Retención de líquidos, que produce hinchazón de brazos y piernas, presión arterial alta o líquido en los pulmones (edema pulmonar). Un aumento repentino de los niveles de potasio en la sangre (hiperpotasiemia) que puede deteriorar el funcionamiento del corazón y puede ser potencialmente mortal. Enfermedad cardíaca y de los vasos sanguíneos (cardiovascular). Huesos débiles e incremento del riesgo de fracturas. Anemia. Disminución del deseo sexual, disfunción eréctil o fecundidad reducida. Daño al sistema nervioso central que puede producir dificultad para la concentración, cambios en la personalidad o convulsiones. Disminución de la respuesta inmune que te hace más vulnerable a la infección. Pericarditis, una inflamación de la membrana con forma de saco que envuelve a tu corazón (pericardio).

En relación a estas consideraciones, Sandoval (ob.cit), precisa que la insuficiencia renal consiste en el deterioro progresivo e irreversible de la función renal. Cuando el filtrado glomerular - filtrado de la sangre en el riñón- cae por debajo del 25 al 35\% empiezan a aumentar la urea y creatinina, pudiendo estar los pacientes relativamente asintomáticos o bien presentando anemia, 


\section{Nivel nutricional y régimen alimenticio de pacientes con insuficiencia renal crónica de un centro de diálisis de Chile}

Vol. 3, núm. 3., (2019)

Mariela del Pilar Bedoya Paucar; Rosa Piedad Morán Asencio; Galo Enrique Estupiñan Vera.

hipertensión arterial, poliuria y nicturia. Al caer el filtrado glomerular cae por debajo del 15\% aproximadamente empiezan a aparecer los signos del síndrome urémico.

Figura $\mathbf{N}^{\circ} 1$ Clasificación de Enfermedad Renal Crónica

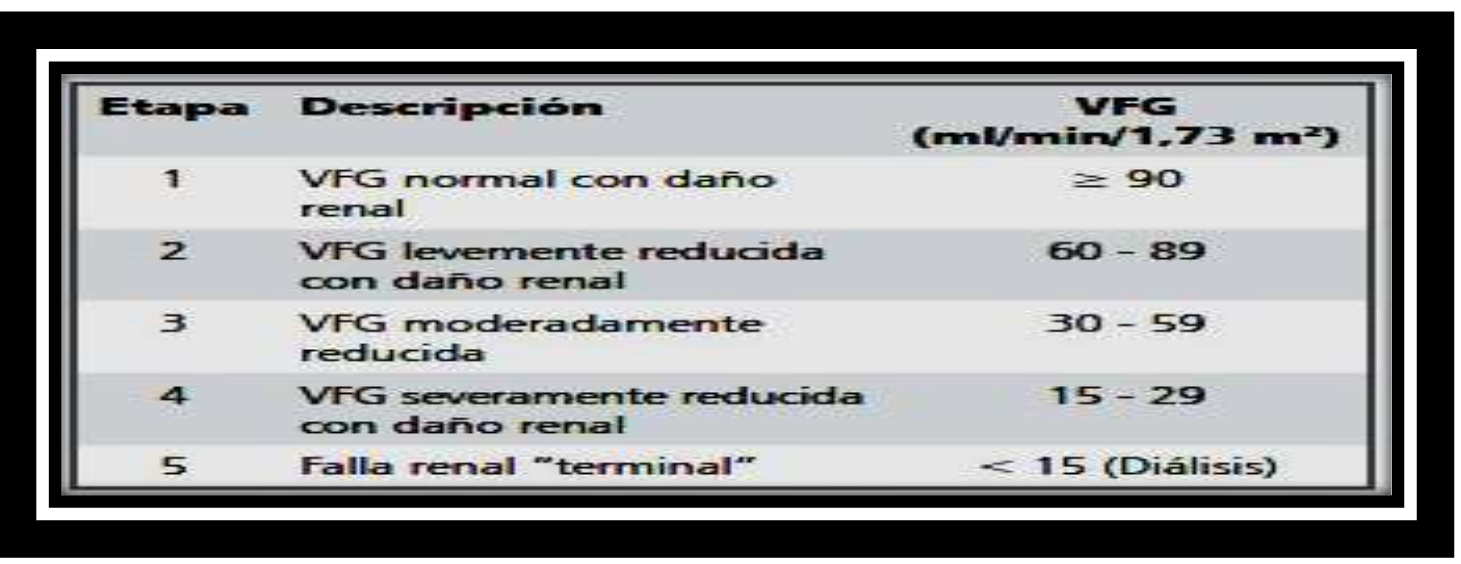

Fuente: Sandoval (ob.cit)

En el mundo se estima que 195 millones de mujeres sufren de ERC con 600 mil muertes por esta causa, situándose como la octava causa de mortalidad para ellas. Esta enfermedad tiene una prevalencia aproximada de $12 \%$ en hombres y de $14 \%$ en mujeres. En Chile, en ellas llega a 14,5\%, mientras que en ellos a 7,4\%. En relación a Chile, se puede indicar que existe una prevalencia estimada en un $14,5 \%$, en mujeres, mientras que los hombres representan el $7,4 \%$. Estos datos, llevan a entender que la Enfermedad Renal Crónica (ERC) es una pérdida progresiva de la función renal en los cuales va disminuyendo la capacidad de eliminar desechos, concentrar la orina, mantener el balance de sustancias en la sangre y de conservar equilibrio de metabolitos y hormonas. (Sandoval, ob.cit, p. 8).

Según lo descrito, el daño de los riñones es irreversible que finalmente requiere tanto diálisis como un trasplante de riñón para poder sobrevivir. Es decir, cuando una persona es 


\section{Nivel nutricional y régimen alimenticio de pacientes con insuficiencia renal crónica de un centro de diálisis de Chile}

Vol. 3, núm. 3., (2019)

Mariela del Pilar Bedoya Paucar; Rosa Piedad Morán Asencio; Galo Enrique Estupiñan Vera. debidamente diagnosticada con insuficiencia renal crónica, diálisis o un trasplante renal para poder vivir. También puedes optar por no someterte a la diálisis ni al trasplante y recibir atención farmacológica para controlar los síntomas con el objetivo de lograr la mejor calidad de vida posible por el tiempo que viva. Los tratamientos no pueden curar la enfermedad renal, pero pueden retrasarla, entre ellos se encuentran: los medicamentos hipertensivos, controlar de azúcar en la sangre, para reducir los lípidos. Dado que, la enfermedad renal crónica aún puede empeorar con el tiempo y conducir a la insuficiencia renal, donde los riñones fallas, siendo determinante el uso de diálisis o un trasplante de riñón como medida final.

Es importante resaltar que los, indicadores de salud pública a nivel mundial y nacional muestran un alarmante y progresivo aumento del número de pacientes con enfermedad renal crónica (ERC), asociado a la elevada prevalencia de patologías como la hipertensión arterial y diabetes mellitus. La ERC puede progresar a insuficiencia renal crónica y tiene un mayor riesgo de enfermedad cardiovascular y mortalidad. Particularmente en Chile, los indicadores epidemiológicos señalan una prevalencia de ERC en la población general de 2,7\%, y un incremento del número de pacientes en hemodiálisis crónica de 12,7 pacientes por millón de personas. (Sandoval ob.cit, p. 12). 


\section{Nivel nutricional y régimen alimenticio de pacientes con insuficiencia renal crónica de un centro de diálisis de Chile}

Vol. 3, núm. 3., (2019)

Mariela del Pilar Bedoya Paucar; Rosa Piedad Morán Asencio; Galo Enrique Estupiñan Vera.

\section{Gráfico $\mathbf{N}^{\circ} 1$ Prevalencia de la Insuficiencia Renal Crónica por Sexo y Edad Chile}

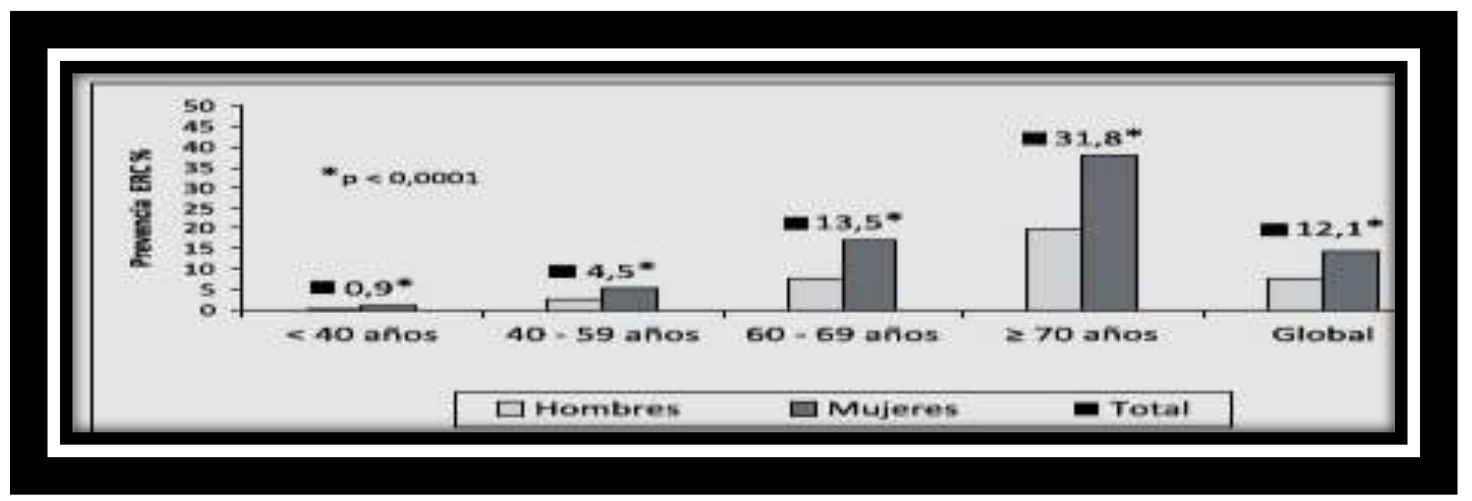

Fuente: Sandoval (ob.cit)

Según los datos presentados, se puede observar que la tendencia aumenta en la medida que la persona se ubica en más de setenta años, aunque su crecimiento tiene un inicio a los sesenta años, para así, entender que en la medida que los individuos llegan a una edad mayor, en ese mismo sentido, los factores de las enfermedades renales crónicas comienzan a tener mayor influencia negativa.

\section{Diálisis en un Centro de Chile}

Cuando los riñones están sanos, limpian su sangre. También producen hormonas que mantienen sus huesos fuertes y su sangre sana. Cuando los riñones fallan, es necesario un tratamiento para sustituir el trabajo que ellos realizaban. A menos que reciba un trasplante de riñón necesitará de un tratamiento llamado diálisis. Existen dos tipos principales de diálisis. Ambos tipos filtran la sangre para eliminar los desechos peligrosos del cuerpo, exceso de sal y agua. La hemodiálisis usa una máquina. A veces es llamada riñón artificial. Es importante, ir en forma frecuente a una clínica especial para recibir el tratamiento varias veces a la semana. La diálisis 


\section{Nivel nutricional y régimen alimenticio de pacientes con insuficiencia renal crónica de un centro de diálisis de Chile}

Vol. 3, núm. 3., (2019)

Mariela del Pilar Bedoya Paucar; Rosa Piedad Morán Asencio; Galo Enrique Estupiñan Vera.

peritoneal usa la membrana que recubre el abdomen, llamada membrana peritoneal, para filtrar la sangre.

La diálisis es un tratamiento para ayudar a filtrar la sangre mediante el cual se filtran los desechos y los líquidos de la sangre para que las personas con falla de los riñones se sientan mejor y puedan llevar una vida normal. Sin embargo, al pensar en diálisis, muchas personas sienten miedo, y el miedo les impide aprender sobre el procedimiento. En realidad, muchos pacientes se sorprenden al descubrir lo bien que funciona la diálisis y lo bien que se sienten con el tratamiento.

Asimismo, la diálisis peritoneal suena como algo complicado, pero en realidad es muy simple. En este tipo de diálisis se usa el revestimiento del abdomen, o barriga, para filtrar la sangre. Este revestimiento se llama membrana peritoneal y actúa como un riñón artificial. Durante el tratamiento se coloca un líquido especial en el abdomen que absorbe los productos de desecho de la sangre cuando esta pasa a través de los pequeños vasos sanguíneos del peritoneo. Luego, se extrae el líquido junto con los desechos. La diálisis peritoneal se hace en la casa del paciente. Muchas personas optan por hacer este tratamiento de noche, mientras duermen.

Según lo planteado, la diálisis puede ser necesaria por diferentes motivos, pero la más frecuente es la incapacidad de los riñones para filtrar adecuadamente los productos de desecho de la sangre (insuficiencia renal). La funcionalidad renal puede disminuir rápidamente (llamada lesión renal aguda o insuficiencia renal aguda), o bien los riñones pueden perder lentamente su capacidad de filtrar los productos de desecho (llamada enfermedad renal crónica o insuficiencia renal crónica). 


\section{Nivel nutricional y régimen alimenticio de pacientes con insuficiencia renal crónica de un centro de diálisis de Chile}

Vol. 3, núm. 3., (2019)

Mariela del Pilar Bedoya Paucar; Rosa Piedad Morán Asencio; Galo Enrique Estupiñan Vera.

En las personas con insuficiencia renal, los médicos recomiendan la diálisis cuando los análisis de sangre muestran que los riñones ya no pueden filtrar adecuadamente los productos de desecho y su acumulación causa problemas. Si se trata de lesión renal aguda, los médicos continúan con la diálisis hasta que los resultados de los análisis de sangre indican que la persona ha recuperado la función renal adecuada. Para las personas con enfermedad renal crónica, la diálisis puede utilizarse como una terapia a largo plazo o como medida temporal hasta que la persona pueda recibir un riñón trasplantado. La diálisis a corto plazo o la de urgencia también pueden utilizarse para eliminar líquidos, determinados fármacos o venenos del organismo.

Tomar la decisión de comenzar un proceso de diálisis de larga duración no es fácil, pues, esta decisión significa un cambio en el estilo de vida de la persona, que incluye el hecho de depender de una máquina para mantenerse con vida. Sin embargo, para la mayor parte de las personas, un programa de diálisis eficaz proporciona una calidad de vida aceptable. La mayoría de las personas sometidas a diálisis pueden seguir una dieta tolerable, tienen una presión arterial normal y evitan la progresión del daño neural, la anemia grave (una disminución del número de glóbulos rojos en la sangre, que transportan oxígeno a las células del organismo) y otras complicaciones peligrosas.

En razón de lo anterior, Pérez, Salas, León y López (ob.cit), precisan que cuando las personas tienen una mala alimentación, es un factor importante de la calidad de vida porque aumenta la tasa de morbilidad y mortalidad reduce la actividad física. La desnutrición, pérdida de peso y posterior aumento de la pérdida de energía de estos pacientes se presenta con fatiga, malestar y agotamiento. Mientras tanto, hay una mayor susceptibilidad a las infecciones como 


\section{Nivel nutricional y régimen alimenticio de pacientes con insuficiencia renal crónica de un centro de diálisis de Chile}

Vol. 3, núm. 3., (2019)

Mariela del Pilar Bedoya Paucar; Rosa Piedad Morán Asencio; Galo Enrique Estupiñan Vera. reservas de proteínas y grasas corporales se encuentran en niveles bajos. Problemas de desnutrición como la anorexia, debido auremia, hipoalbuminemia y disminución de la ingesta de proteína, parece estar exacerbada por la presencia de síntomas depresivos. De allí, la importancia de considerar durante la realización del tratamiento por diálisis, un efectivo régimen alimenticio que le proporcione al paciente el nivel nutricional requerido para la realización de sus actividades diarias.

\section{Nivel Nutricional y Régimen Alimenticio}

La alimentación es importante porque ayuda a mejorar la salud de los seres humanos. Los nutrientes pasan por los diferentes sistemas orgánicos de las personas extrayendo las vitaminas y minerales necesarios. El resto del alimento empieza a ser digerido y transformado por diversos órganos que cumplen dicha función. Uno de estos sistemas es el urinario. Este aparato urinario comprende los riñones, la uretra, la vejiga urinaria y los uréteres. Los riñones forman parte fundamental de este sistema y es un parámetro fundamental para la calidad de vida de las personas. Los riñones son un órgano par de color pardo rojizo que se hallan en la parte posterior del peritoneo, a ambos lados de la columna vertebral. Tienen una superficie lisa que presenta una profunda depresión en su borde interno denominada hilio renal. Cachofeiro, Lahera, \& Tresguerres, 1999, (citado por Villao, Vásquez, Pérez y Padovani 2019).

De allí, que debe hablar una relación terapéutica que permita tomar la forma de una 'participación mutua', donde el paciente tiene que participar activamente en el tratamiento y cumplir con las instrucciones médicas. Adicionalmente, el paciente tiene que adquirir conocimientos pertinentesal manejo de la enfermedad, recibir apoyo y formación o enseñanza de 


\section{Nivel nutricional y régimen alimenticio de pacientes con insuficiencia renal crónica de un centro de diálisis de Chile}

Vol. 3, núm. 3., (2019)

Mariela del Pilar Bedoya Paucar; Rosa Piedad Morán Asencio; Galo Enrique Estupiñan Vera.

profesionales de la salud para lograr una mejor calidad de vida relacionada con la salud. En este

punto, vale la pena mencionar la estrecha colaboración de los miembros de equipos multidisciplinario de tratamiento (nutricionistas, psicólogos, técnicos de máquinas). También, la participación de los pacientes en las redes de apoyo o actividades de rehabilitación y participación en un programa de actividad física o educativa.

Como no existe un patrón único para la nutrición en pacientes en diálisis, el diagnóstico de malnutrición debe apoyarse en varios parámetros en un paciente concreto. El parámetro nutricional más importante es el contenido proteico corporal, por lo tanto, los pacientes deben seguir una dieta rica en proteínas. "Los requerimientos proteicos de los pacientes en diálisis parecen ser mayores que en los individuos normales...la recomendación proteica es de 1,2 g/Kg/día como mínimo. La calidad de la proteína también es importante..." (Riella \& Martins, (citado por Torres, Romo y Gómez 2019). Ahora bien, los alimentos ricos en proteínas generalmente tienen alto contenido de fósforo y potasio; en los pacientes en tratamiento de diálisis el aumento en los niveles de fósforo es perjudicial para su salud, ya que producen calcificación en las venas y arterias.

En conclusión, la dieta de un paciente con tratamiento diálisis debe estar basada en alimentos ricos en proteínas y bajos en fósforo. Además, debe hacerse un seguimiento nutricional, bioquímico y antropométrico, lo que hace de la historia clínica, de los exámenes de laboratorio y del examen físico un instrumento de medición y control. Este seguimiento se realiza para garantizar la calidad de vida del paciente y alargar su esperanza de vida. "Para prevenir la desnutrición, es importante efectuar el seguimiento del estado nutricional. Por lo tanto, los 


\section{Nivel nutricional y régimen alimenticio de pacientes con insuficiencia renal crónica de un centro de diálisis de Chile}

Vol. 3, núm. 3., (2019)

Mariela del Pilar Bedoya Paucar; Rosa Piedad Morán Asencio; Galo Enrique Estupiñan Vera.

pacientes deben ser evaluados al comienzo del programa de diálisis y cada cuatro a seis meses" Riella \& Martins, 2004. (citado por Torres, Romo y Gómez ob.cit ).

Los pacientes con insuficiencia renal crónica en tratamiento de diálisis requieren cuidado complementario en el hogar, debido a que en el centro de salud reciben tratamiento médico, psicológico y social; pero estos son procedimientos que deben ser acompañados de la labor que se realiza en casa, con el estilo de vida, con las actividades cotidianas y con la alimentación. La alimentación del paciente con IRC debe tener un alto contenido proteico y bajo contenido en fósforo, por lo que la presente investigación supone que una dieta con estas características se relaciona positivamente con el estado nutricional del paciente con tratamiento de diálisis.

Cabe destacar que, durante la realización de la diálisis, se presentan diferentes efectos colaterales, citados por Opazo, Razeto y Huanca (2016), precisa los siguientes: La Toxicidad Urémica: se debe a la escasa o nula eliminación renal de solutos de bajo, mediano y alto peso molecular; siendo las moléculas de menor tamaño las de pequeño peso molecular a las que se les ha atribuido un papel fundamental en la sintomatología de la uremia. En los pacientes en diálisis el aclaramiento de estos solutos está íntimamente relacionado con: a) la superficie y eficacia depuradora de la membrana de diálisis (expresada como el coeficiente de transferencia de masa de urea (KoA); b) los flujos de sangre y líquido de diálisis, y c) fundamentalmente del tiempo de duración de la sesión de diálisis.

Asimismo, Hiperkalemia en HD: El Potasio $(\mathrm{K}+)$ es el catión de mayor presencia en el espacio intracelular (98\%) y en el espacio extracelular (2\%). La homeostasis del K+ resulta de la excreción renal y la regulación de su transporte entre los compartimientos intra y extracelular, los 


\section{Nivel nutricional y régimen alimenticio de pacientes con insuficiencia renal crónica de un centro de diálisis de Chile}

Vol. 3, núm. 3., (2019)

Mariela del Pilar Bedoya Paucar; Rosa Piedad Morán Asencio; Galo Enrique Estupiñan Vera.

principales reguladores son la insulina, las catecolaminas, el estado ácido base y la osmolaridad

sérica. Del K+ ingerido diariamente se excreta entre el 80-95\% vía urinaria y 5-20\% por la vía

fecal. Cuando el índice de filtración glomerular (IFG) desciende a menos de $15 \mathrm{ml} / \mathrm{minuto}$ disminuye la excreción renal de $\mathrm{K}+$, resultando esencial la intervención nutricional con énfasis de la reducción de la ingesta de potasio en la dieta. En HD se estima como valores deseables de potasio sérico entre 3,5 y 5,0 mEq/L. Los pacientes con escasa o ninguna función renal, sobre todo los anúricos son propensos a desarrollar Hiperkalemia, en casos severos, puede precipitar arritmias fatales.

La concentración de $\mathrm{K}+$ en el líquido de diálisis varía entre 1-3 mEq/L, en función de la situación clínica del paciente y los niveles séricos de $\mathrm{K}+$, en caso de hiperkalemia con significado clínico se preferirá usar concentrados con $\mathrm{K}+$ bajo; y de baño con concentraciones incluso más altas que $3 \mathrm{mEq} / \mathrm{L}$. Es importante mencionar además que podemos optimizar las pérdidas de $\mathrm{K}+$ por las heces y para ello debemos asegurar que los pacientes tengan deposiciones diarias, incluso podemos utilizar resinas de intercambio. Frente a un paciente con hiperkalemia persistente en HD debemos hacer un interrogatorio con énfasis en: ingesta oral de $\mathrm{K}+$, uso de fármacos (como IECA, ARA2), pérdidas sanguíneas por deposiciones, estado de acidosis entre otras.

De igual manera, se encuentra Hipervolemia y Sodio en HD: Producto de la pérdida de la función renal y la incapacidad del organismo de poder regular su medio interno, en los pacientes con ERC-T en HD, tiende a producirse ganancia de volumen con incremento del agua corporal total tanto del compartimento intracelular como del extracelular, expresándose en síntomas y signos clínicos como disnea, ortopnea, edema, ingurgitación yugular, hipertensión arterial 


\section{Nivel nutricional y régimen alimenticio de pacientes con insuficiencia renal crónica de un centro de diálisis de Chile}

Vol. 3, núm. 3., (2019)

Mariela del Pilar Bedoya Paucar; Rosa Piedad Morán Asencio; Galo Enrique Estupiñan Vera.

(volumen dependiente) e incluso, situaciones graves de edema pulmonar agudo y compromiso del sistema nerviosos central.

El Na+ en el baño de diálisis oscila entre 135-145 mEq/L, y la concentración utilizada va a depender de las condiciones clínicas del paciente, ya sea del agua corporal total, de la presión arterial y sobretodo de la ganancia de peso interdiálisis. Durante la HD hay que extraer el agua y la sal acumuladas entre dos sesiones de diálisis. El componente de pérdida difusiva del $\mathrm{Na}+$ es más importante durante la diálisis con baños hiposódicos; sin embargo, ello conlleva una deshidratación extracelular y sobrehidratación intracelular, no deseables. Cuando el Na+ del baño es similar al del agua plasmática el proceso difusivo cesa, la pérdida de agua y sal se realizan por mecanismos convectivos. El Na+ es importante en la estabilidad cardiovascular es necesario establecer un balance dialítico exacto para evitar efectos adversos.)

El tipo de alimento consumido influye también en el proceso inflamatorio del paciente en HD, porque el aumento del consumo de los alimentos procesados sometidos a altas temperatura por tiempo prolongado desarrolla productos finales de Glicación (AGEs) y productos finales de Lipoxidación (ALEs) como consecuencia de la reactividad de los H. de C., proteínas, lípidos y otros componentes. La ingesta continuada de AGEs y ALEs contribuye al acúmulo corporal de estos productos e influyen negativamente en el sistema inmunológico, la respuesta inflamatoria y la resistencia a la enfermedad a través de la interacción con los denominados receptores de AGEs (RAGE).

Los cambios en el estilo de vida tales como menos actividad física, estrés y la transición desde el consumo de alimentos naturales no procesados a alimentos procesados ricos en calorías 


\section{Nivel nutricional y régimen alimenticio de pacientes con insuficiencia renal crónica de un centro de diálisis de Chile}

Vol. 3, núm. 3., (2019)

Mariela del Pilar Bedoya Paucar; Rosa Piedad Morán Asencio; Galo Enrique Estupiñan Vera.

y alimentos modificados, con una reducción significativa en la ingesta de fibra vegetal, antioxidantes, ácidos grasos poliinsaturados (PUFAs) de la serie Omega 3, la abundante ingesta de ácidos grasos saturados y ácidos grasos trans. El gran consumo de alimentos de alto índice glicémico (IG): productos azucarados y con elevado contenido en almidones. Los procesos industriales que tienen como objetivo hacer que los alimentos sean más seguros, más aromáticos y con más color, tales como el calentamiento (reacción de Maillard), la irradiación y la ionización, todos ellos en combinación con la sobrenutrición, contribuyen de manera significativa a la producción y a la exposición y acumulación en el cuerpo de AGEs y ALEs.

Los pacientes con enfermedades renales crónicas moderada tienen concentraciones aumentadas hasta 5 veces de radicales libres de glicación en el plasma y en los pacientes con ERCT con terapia en PD alrededor de 18 veces y aquellos que están en HD aumenta hasta 40 veces. En enfermedades tales como la DM, enfermedad vascular y ERC; todas ellas responden con una considerable reducción en los marcadores de inflamación y disfunción vascular, cuando se suministra una dieta baja en AGEs. (20). Las medidas dietéticas para reducir las AGEs/ALEs

Pérdidas de Nutrientes en el Dializado: Pueden ser un factor importante de desnutrición, se pierde principalmente aminoácidos (a.a.), proteínas y vitaminas hidrosolubles. En cada sesión de HD de bajo flujo se pierde 5 a $8 \mathrm{~g}$ de a.a. libres y 4 a $5 \mathrm{~g}$ de a.a. ligados. Las variaciones del área de superficie de las diferentes membranas dializadoras y de las velocidades del flujo sanguíneo podrían influir en las pérdidas de a.a. en el dializado. La reutilización de los filtros y su procesamiento con sustancias químicas como el hipoclorito de $\mathrm{Na}$ pueden provocar un aumento en las pérdidas proteicas debido al aumento de la permeabilidad de las membranas. La sangre 


\section{Nivel nutricional y régimen alimenticio de pacientes con insuficiencia renal crónica de un centro de diálisis de Chile}

Vol. 3, núm. 3., (2019)

Mariela del Pilar Bedoya Paucar; Rosa Piedad Morán Asencio; Galo Enrique Estupiñan Vera.

retenida en el filtro se estima entre 5 a $10 \mathrm{ml}$ lo que puede corresponder a una pérdida proteica de 0,6 a 1,4 g por sesión de diálisis, las cuales pueden verse aumentada por filtros obstruidos o con fugas.

Las Pérdidas de Vitaminas Hidrosolubles en Diálisis: no son grandes, esto se debe al hecho que las concentraciones plasmáticas de estas son pequeñas y sus pesos moleculares son elevados. Las mayores pérdidas son de vits. B1, B2, y B6, B12, se Vit C y Ac. Fólico. Las pérdidas de Vits. Hidrosolubles en el dializado pueden compararse con las que tienen normalmente por medio de la orina. La suplementación oral es necesaria además por la baja ingesta de verduras y frutas crudas, y el remojo y la cocción de los alimentos que utiliza para reducir su contenido de $\mathrm{K}+\mathrm{y} \mathrm{P}+$.

Mal Nutrición y Balance Nitrogenado en Diálisis: La malnutrición calórico-proteica y la emaciación son comunes en el paciente en ERC-T y aunque los procedimientos asociados a la terapia dialítica, como bioincompatibilidad de la membrana y pérdida de nutrientes, pueden contribuir a la malnutrición, ésta es común incluso antes del inicio de la terapia de reemplazo renal (TRR). De causa multifactorial, comprende alteraciones en el metabolismo proteico energético, alteraciones hormonales e ingesta alimentaria deficiente, debido principalmente a la anorexia, náuseas y vómitos asociados a estados de toxicidad urémica. Varias enfermedades como la DM y la enfermedad vascular difusa (caquexia vascular), así como las afecciones superpuestas (pericarditis, infecciones, insuficiencia cardiaca congestiva) pueden contribuir a la desnutrición.

La caquexia en enfermedades renales crónica está caracterizada por el catabolismo proteico. La síntesis de proteínas se mantiene sin cambio mientras que la degradación proteica está aumentada, mecanismo generado a través de la activación del sistema ubiquitín proteasoma. Las 


\section{Nivel nutricional y régimen alimenticio de pacientes con insuficiencia renal crónica de un centro de diálisis de Chile}

Vol. 3, núm. 3., (2019)

Mariela del Pilar Bedoya Paucar; Rosa Piedad Morán Asencio; Galo Enrique Estupiñan Vera.

complicaciones asociadas a esta enfermedad incluyendo acidosis metabólica, resistencia a la insulina, inflamación y aumento de la producción de glucocorticoides y angiotensina II, todos activan el sistema ubiquitín proteasoma provocando la degradación de proteínas musculares

Al tomar en consideración los diferentes efectos que se agregan en el paciente dializado, es importante considerar que los mismos tienen riesgo de desnutrición por ingesta inadecuada de nutrientes, pérdida proteica a través del dializado, inflamación, acidosis metabólica o cambios hormonales. Se estima una malnutrición proteico-energética de aproximadamente el 20-50\% de estos pacientes, lo que hace que mantener el equilibrio nutricional sea un objetivo prioritario del plan de cuidados de los mismos. La valoración continua del estado nutricional permitirá controlar el estado del paciente y detectar precozmente las posibles desviaciones nutricionales, al mismo tiempo que servirá para hacer prevención de las complicaciones de la insuficiencia renal y del estado general de salud del paciente asociadas a la desnutrición.

En esta misma dirección Flores, Alvo, Borja, Morales, Vega y otros (2015), destacaron en su estudio que la malnutrición es un problema que afecta a un 40-50\% de los pacientes que tienen enfermedad renal crónica con tratamiento de hemodiálisis, situación que provoca infecciones, mala cicatrización de heridas, pérdida muscular, e incluso un incremento de la mortalidad de estos pacientes. Esta población se clasifica en riesgo de desnutrición, debido a la pérdida de nutrientes en la dializada y mala educación sanitaria, aumentando el riesgo con la edad avanzada y dosis inadecuada de hemodiálisis que pueden provocar hipoalbuminemia. 


\section{Nivel nutricional y régimen alimenticio de pacientes con insuficiencia renal crónica de un centro de diálisis de Chile}

Vol. 3, núm. 3., (2019)

Mariela del Pilar Bedoya Paucar; Rosa Piedad Morán Asencio; Galo Enrique Estupiñan Vera.

De acuerdo con los diferentes problemas adversos que registra el paciente de diálisis en Chile, para lograr una mejor calidad de vida es importante considerar lo citado por F lores, Alvo, Borja, Morales, Vega y otros (ob.cit), incorporar lo siguiente:

Proteínas: sirven para la construcción y renovación de las células y tejidos. Son imprescindibles para el correcto funcionamiento de nuestro cuerpo. Durante la diálisis se pierden proteínas, por lo que debe reponerlas con la alimentación. La ingesta de proteínas recomendada para un paciente de hemodiálisis es de 1,0-1,2 g/kg de peso cada día. Aproximadamente el 50\% deben ser de alto valor biológico.

Hidratos de Carbono: Son la principal fuente de energía del organismo. Existen dos tipos de hidratos de carbono: Simples (o de absorción rápida): azúcar, miel, caramelos, bollería, pasteles, chocolates. Complejos (o de absorción lenta): arroz, pasta, patata, cereales, legumbres. Es mejor tomar hidratos de carbono complejos y evitar los simples que favorecen subidas de colesterol, triglicéridos, obesidad, entre otros.

Grasas: Son la principal reserva energética del cuerpo. Existen dos tipos de grasas: Saturadas: se encuentran en alimentos de origen animal (embutidos, huevo, lácteos enteros, mantequilla, etc.). Su consumo está relacionado con las enfermedades cardiovasculares. Insaturadas: se encuentran en pescados y alimentos de origen vegetal (aceite de oliva, de semillas, margarinas vegetales, etc.). Su consumo protege de las enfermedades cardio-vasculares.

Vitaminas: Tienen una función reguladora, actuando en diferentes acciones del metabolismo. Son esenciales para la vida y debe incluirlas en su alimentación. En cada sesión de 


\section{Nivel nutricional y régimen alimenticio de pacientes con insuficiencia renal crónica de un centro de diálisis de Chile}

Vol. 3, núm. 3., (2019)

Mariela del Pilar Bedoya Paucar; Rosa Piedad Morán Asencio; Galo Enrique Estupiñan Vera.

hemodiálisis se pierde cierta cantidad de vitaminas hidrosolubles. Existen preparados vitamínicos que en caso necesario serán pautados por su nefrólogo. Por otra parte, el riñón es el encargado de activar la vitamina D procedente de los alimentos, para así, poder absorber el calcio de la comida. Cuando el riñón no funciona, no se activa esta vitamina y no se puede utilizar el calcio presente en los alimentos. Existen medicamentos que aportan vitamina D activa para mejorar la absorción del calcio.

Minerales: Su presencia en la dieta es necesaria para una correcta alimentación. Sodio: Es el principal componente de la sal de mesa. Los riñones son los encargados de eliminarlo. Si no funcionan se acumula en el organismo, provocando retención de agua. Ese líquido acumulado puede producir hinchazón (piernas, párpados, etc.), tensión arterial elevada, insuficiencia cardiaca y edema de pulmón. Como norma general, al realizar diálisis, debe seguir una dieta pobre en sal (entre 1.300 y $1.700 \mathrm{mg}$. de sodio al día).

Potasio: Es un mineral necesario para el funcionamiento del sistema nervioso y muscular. Los riñones son los encargados de eliminar el exceso de potasio del organismo. Cuando no funcionan se acumula en la sangre. Su exceso en el organismo produce: hormigueos, calambres, debilidad muscular, arritmias e incluso parada cardiaca, por lo que puede convertirse en el componente más peligroso de la alimentación. El potasio se encuentra en la mayoría de los alimentos, pero sobre todo en frutas, verduras, legumbres y frutos secos. Para su control, como es un mineral soluble en agua, parte del potasio de algunos alimentos (patatas, verduras, legumbres, etc.) lo puede eliminar haciendo remojo y doble cocción. 


\section{Nivel nutricional y régimen alimenticio de pacientes con insuficiencia renal crónica de un centro de diálisis de Chile}

Vol. 3, núm. 3., (2019)

Mariela del Pilar Bedoya Paucar; Rosa Piedad Morán Asencio; Galo Enrique Estupiñan Vera.

Calcio y Fósforo: Son el principal componente de los huesos. Ambos minerales trabajan juntos, en equilibrio. Un exceso de fósforo en la sangre, produce una salida de calcio los huesos. Así, los huesos se descalcifican, se hacen más frágiles y se pueden fracturar. El exceso de calcio y fósforo en sangre, forma pequeños depósitos minerales en el organismo. Según donde se localicen estos depósitos, puede haber diferentes problemas de salud.

Agua: El agua es esencial para la vida y el buen funcionamiento del organismo. Cuando se realiza tratamiento de hemodiálisis la cantidad de orina desciende e incluso puede dejar de orinar. Así, entre dos sesiones de diálisis, aumenta el peso corporal debido a la acumulación de líquidos. Esta ganancia de peso debe ser de 1,5 a 2,5 kg. Como máximo.

Figura $\mathbf{N}^{\circ} 2$ Régimen Alimenticio de Pacientes en Diálisis

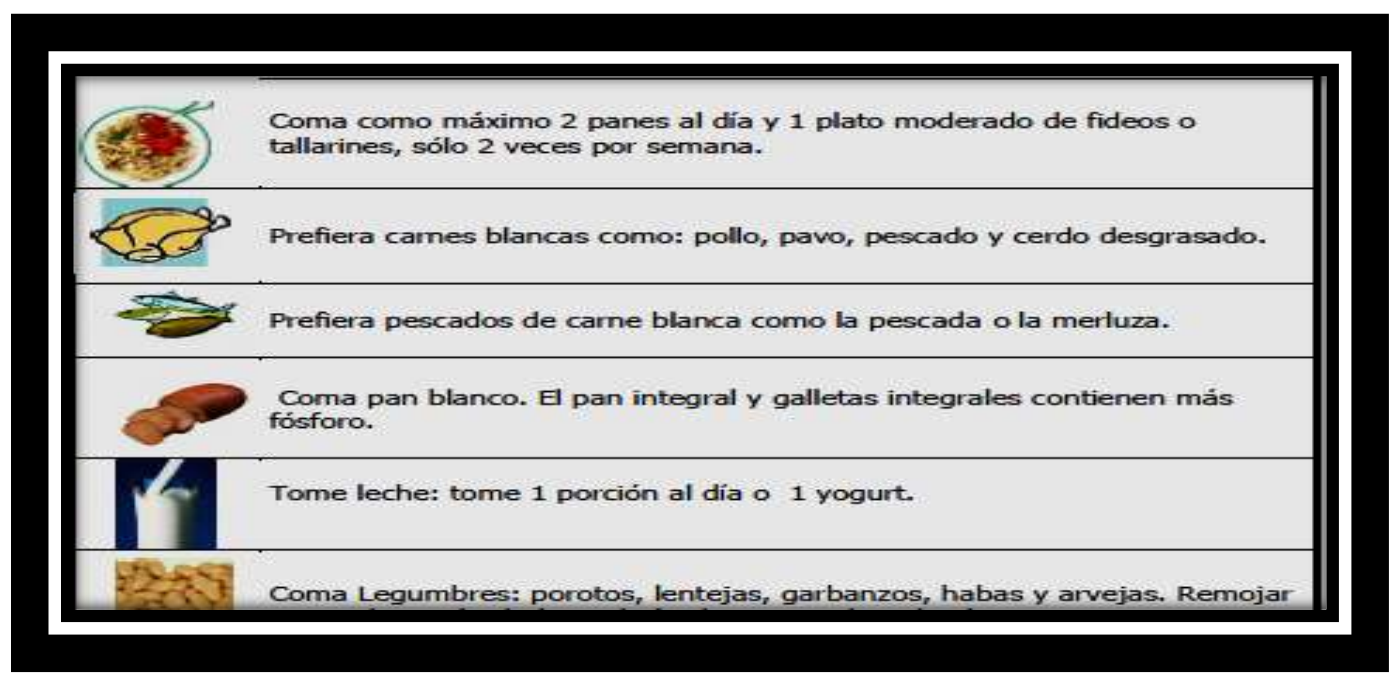

Fuente: Flores, Alvo, Borja, Morales, Vega y otros (ob.cit)

Estos últimos autores, complementan la necesidad de lograr incrementar el nivel proteico que los pacientes debe tener, para lo cual, es relevante combinar en cada comida una serie de 


\section{Nivel nutricional y régimen alimenticio de pacientes con insuficiencia renal crónica de un centro de diálisis de Chile}

Vol. 3, núm. 3., (2019)

Mariela del Pilar Bedoya Paucar; Rosa Piedad Morán Asencio; Galo Enrique Estupiñan Vera.

alimentos que le permitirán tener el régimen alimenticio ajustado a las posibles situaciones manifestadas durante el tratamiento de diálisis.

Figura $\mathbf{N}^{\circ} 3$ Pirámide de Diálisis

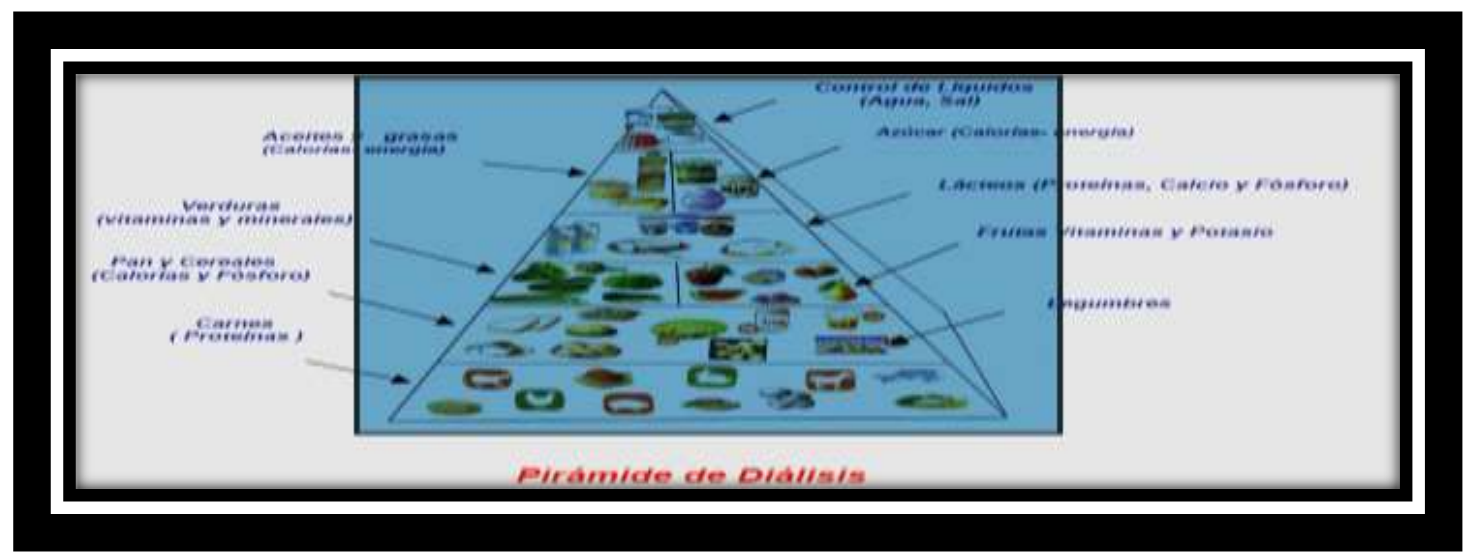

Fuente: Flores, Alvo, Borja, Morales, Vega y otros (ob.cit)

\section{Conclusiones}

Los aspectos citados a continuación, se encuentran relacionados con las valoraciones que el investigador precisa desde su propia percepción, donde las fuentes teóricas han sido básicas para finalmente expresar las ideas generales del hecho investigativo.

El paciente con insuficiencia renal crónica que recibe tratamiento con diálisis, suele sufrir cambios psicológicos, sociales, en su estilo de vida y también en su vida espiritual, que no sólo le afectan a él, sino a toda su familia. Es por este motivo, que es trascendental en la calidad de vida de este tipo de pacientes, que tengan una alimentación adecuada y balanceada, donde la combinación de los diferentes alimentos, se haga de manera efectiva para reducir aquellas posibles 


\section{Nivel nutricional y régimen alimenticio de pacientes con insuficiencia renal crónica de un centro de diálisis de Chile}

Vol. 3, núm. 3., (2019)

Mariela del Pilar Bedoya Paucar; Rosa Piedad Morán Asencio; Galo Enrique Estupiñan Vera. situaciones relacionadas con algunas enfermedades desencadenantes debido a la misma enfermedad preexistente.

Es importante acotar que, el paciente chileno, que asiste al tratamiento de diálisis, se puede indicar que ha producido un efecto mayor sobre la duración de la sobrevida de estos pacientes. Sin embargo, en sistemas cada vez más influenciados por el aspecto económico y el alto costo de los tratamientos, en algunas oportunidades se pone en duda la justificación de los mismos y queda aún pendiente dilucidar su verdadero impacto sobre otro aspecto fundamental como es la calidad de vida del paciente con insuficiencia renal crónica.

Como consecuencia existe un interés creciente en el desarrollo de metodologías para cuantificar el efecto tanto de la patología, como de las medidas terapéuticas aplicadas sobre esta calidad de vida. Estas metodologías varían desde aquellas basadas en la apreciación objetiva de un observador externo, hasta las que toman en cuenta al paciente como fuente subjetiva del impacto provocado por la enfermedad en su vida cotidiana. Esta última forma, en la cual el propio paciente es la fuente de información, se considera hoy como la verdaderamente válida.

Debido que, el paciente con insuficiencia renal crónica en diálisis, suele sufrir cambios psicológicos, sociales, en su estilo de vida y también en su vida espiritual, que no sólo le afectan a él, sino a toda su familia. Algunas personas aceptan el hecho de tener una enfermedad renal y que necesitarán tratamiento para el resto de sus vidas. Otros en cambio, pueden tener problemas para ajustarse a los desafíos que la enfermedad implica. Durante el curso de la enfermedad, habitualmente afloran sentimientos de culpa, negación, rabia, depresión, frustración que son difícilmente comprendidos y aceptados por el paciente y sus familiares. Es muy importante saber 


\section{Nivel nutricional y régimen alimenticio de pacientes con insuficiencia renal crónica de un centro de diálisis de Chile}

Vol. 3, núm. 3., (2019)

Mariela del Pilar Bedoya Paucar; Rosa Piedad Morán Asencio; Galo Enrique Estupiñan Vera.

que es posible desarrollar nuevas capacidades y habilidades que permitan lograr una mejor calidad de vida. Así como también es posible obtener ayuda para asumir la enfermedad y su tratamiento.

Otro aspecto de interés, durante el tratamiento de diálisis que registran los pacientes, es la desnutrición como resultado a la pérdida de proteína y minerales esenciales para mantener su estatus de vida. Es allí, donde se destaca la necesidad de mantener un régimen alimenticio caracterizado por la combinación de proteínas, hidratos de carbono, minerales, entre otros elementos, que le ofrezcan los nutrientes que requieren para lograr un nivel de nutrición ajustada a las condiciones físicas u orgánicas caracterizadoras en cada paciente. Además, es importante continuar con investigaciones enmarcadas en la alimentación, con el fin de dar mayores informaciones capaces de mejorar la calidad de vida de las personas que asisten al tratamiento de diálisis.

\section{Referencias Bibliográficas}

Flores, J, Alvo, M, Borja, H, Morales, J, Vega, J, Zuñiga, C, Muiller, H y Munzenmayer, J. (2015). Enfermedad Renal Crónica. Médica de Chile, 137(1), 137-177.

Pérez Rodríguez, L., Espinosa Sánchez, N., López Contreras, N., \& Pesantes Merchán, D. (2019). Nutrición: Tratamiento para la gastritis. RECIMUNDO, 3(2), 120-137.

Ruiz, A. (2017). Introducción a los Métodos. Caracas: Universidad Central de Venezuela.

Sandoval, V. (2019). Guía de Alimentación en Pacientes con Insuficiencia Renal. Gipuzkoa: Hospital Universitario Donostia.

Uribe, T. (2019). Análisis e Interpretación. México: Trillas.

Villao, A, Vásquez, A, Pérez, N y Padovani, A. (2019). La sepsis como causa de daño renal. RECIMUNDO, 3(2), 628-650. 